\title{
Cell-Based, High-Content Screen for Receptor Internalization, Recycling and Intracellular Trafficking
}

\author{
R.N. Ghosh"1, Y.-T. Chen"1, R. DeBiasio', R.L. DeBiasio', B.R. Conway' ${ }^{2}$ L.K. Minor ${ }^{2}$ \\ and K.T. Demarest ${ }^{2}$ \\ ${ }^{1}$ Cellomics ${ }^{\mathrm{TM}}$ Inc., Pittsburgh, PA, USA, and ${ }^{2}$ R.W. Johnson Pharmaceutical Research \\ Institute, Raritan, NJ, USA
}

BioTechniques 29:170-175 (July 2000)

\begin{abstract}
A variety of physiologically important receptors are internalized and then recycled back to the plasma membrane by the endocytic recycling compartment. These include the transferrin receptor and many G-protein coupled receptors (GPCRs). The internalization of GPCRs is a result of agonist stimulation. A cell-based fluorescent imaging assay is described that detects and quantifies the presence of fluorescently labeled receptors and macromolecules in the recycling compartment. This High Content Screening application is conducted on the ArrayScan ${ }^{\circledR}$ II System that includes fluorescent reagents, imaging instrumentation and the informatics tools necessary to screen for compounds that affect receptor internalization, recycling and GPCR activation. We demonstrate the Receptor Internalization and Trafficking application by quantifying (i) the internalization and recycling of the transferrin receptor using a fluorescently labeled ligand and (ii) the internalization of a physiologically functional model GPCR, a GFP-parathyroid hormone receptor chimera. These assays give high signal-to-noise ratios, broad dynamic ranges between stimulated and unstimulated conditions and low variability across different screening runs. Thus, the Receptor Internalization and Trafficking application, in conjunction with the ArrayScan II System, forms the basis of a robust, information-rich and automated screen for GPCR activation.
\end{abstract}

\section{INTRODUCTION}

High Content Screening (HCS) is a new, whole cell-based screening approach that generates information-rich data regarding the effects of potential drug candidates on multiple cellular targets from a cell-by-cell or population basis $(12,13)$. HCS can be performed on the ArrayScan II System (Cellomics $^{\mathrm{TM}}$, Pittsburgh, PA, USA), a high-resolution imaging and analysis platform that quantifies the spatial and temporal distributions of multiple fluorescently labeled cell constituents inside living cells. Changes in the distribution of fluorescent cellular markers over space and time are automatically analyzed using sophisticated image-based algorithms. Optimized protocols and combinations of validated fluorescent reagents are provided in the HitKit ${ }^{\mathrm{TM}}$ series of screening kits (Cellomics) to facilitate HCS assays, with each kit focusing on specific cellular activities and targets.

This paper describes the practical application of the ArrayScan II System to screen for compounds that affect the internalization, recycling and intracellular trafficking of the transferrin receptor (TfR) using the Receptor Internalization and Trafficking application and the Transferrin Receptor HitKit. This same application was used to quantify the activation of the parathyroid hormone receptor (PTHR).

Integral membrane proteins and macromolecules that are endocytosed at the cell surface have different intracellular itineraries and fates $(14,19)$. Endocytosed receptors that recycle back to the plasma membrane traffic through a compartment known as the endocytic-recycling compartment (ERC) (Figure 1). In many cell types $(14,19)$, the ERC containing fluorescently labeled receptors appears as a fluorescent spot in a peri-nuclear location $(7,10,17)$. Many physiologically important macromolecules traffic through or associate with the ERC, including ( $i$ ) receptors that constitutively internalize and then recycle such as the TfR and the low-density lipoprotein receptor (19); (ii) many GPCRs that are internalized following agonist stimulation and desensitization and then recycle back to the plasma membrane by way of the ERC during resensitization $(2,6,16,25)$; (iii) various lipids $(17,20)$; (iv) glycosylphosphatidylinositol-anchored proteins (18); (v) cholesterol and analogs (21); and (vi) other trafficking-related proteins $(3,8,10,15,27)$. 
An assay to quantify internalization, recycling or association with the ERC can be used to screen for and characterize compounds that affect many physiological processes. Because intracellular trafficking involves the redistribution of macromolecules in cells, it is best quantified by a high-resolution, cellular-imaging approach that can discern subcellular events and targets. TfR is used to characterize this internalization and recycling pathway because its ligand, Tf, stays bound to the receptor during its intracellular trafficking itinerary (19). Fluorescently labeled Tf can be used to monitor TfR internalization, recycling and also to determine the specificity of compounds that affect association of other macromolecules with the ERC. For example, compound treatment caus- ing reduced association of a GPCR with the ERC could be caused by its being either an antagonist or a general endocytosis inhibitor. Assaying its effect on the trafficking of the TfR will help distinguish between these possibilities.

Cellomics has developed an HCS application that detects the presence of internalized fluorescently labeled receptors in the ERC (2) and optimized fluorescent reagents for use with the application to assay for $\mathrm{Tf}$ trafficking and association with the ERC. We demonstrate the use of the TfR HitKit and the Receptor Internalization and Trafficking application in screening assays for TfR internalization and recycling. We also describe our use of this application to assay for the activation and internalization of PTHR.

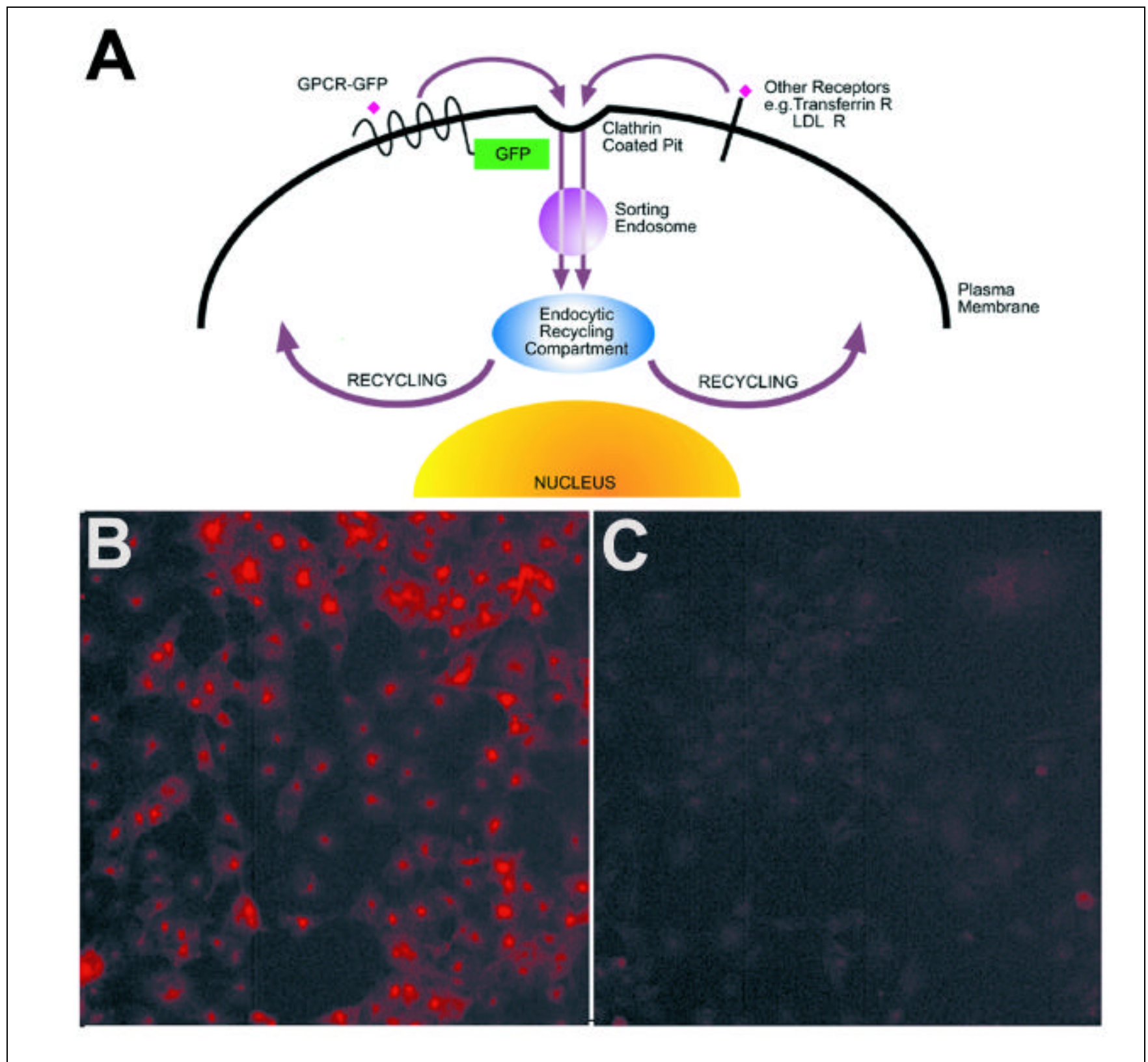

Figure 1. Receptor internalization and recycling pathways. (A) Schematic of cell surface receptor internalization and recycling pathways; (B) Internalization of the TfR. Because transferrin remains bound to its receptor during its post-endocytic intracellular trafficking route, fluorescently labeled Tf serves as a useful marker for quantifying $\mathrm{Tf}$ internalization and recycling of its receptor. COS cells grown on a microplate were incubated with $20 \mu \mathrm{g} / \mathrm{mL}$ Alexa 546 -conjugated Tf for $45 \mathrm{~min}$, then fixed and imaged on the ArrayScan II. Bright peri-nuclear staining typical of internalized receptor in the ERC was observed. (C) Recycling of the TfR. After incubation with AxTf, cells were incubated in nonfluorescent chase medium for $60 \mathrm{~min}$ before fixation and imaging. Faint staining was observed, consistent with successful chasing of AxTf from the ERCs. 


\section{MATERIALS AND METHODS}

The key components in the TfR HitKit are diferric transferrin conjugated to the fluorophore, Alexa ${ }^{\mathrm{TM}} 546$ (AxTf; excitation and emission maxima at 554 and $570 \mathrm{~nm}$ ), and the fluorescent nucleic acid stain, Hoechst 33342 (excitation and emission maxima at 350 and $461 \mathrm{~nm}$ ). These can be used to assay for either TfR internalization or TfR trafficking from the ERC back to the plasma membrane.

In these assays, COS- 1 cells were plated in 96-well, optically clear-bottomed microplates (Packard Instrument, Meriden, CT, USA) at $10^{4}$ cells/well, $18 \mathrm{~h}$ before the experiment in McCoy5A medium with 5\% fetal bovine serum (FBS) and $4 \mu \mathrm{M}$ deferoxamine mesylate (Sigma, St. Louis, MO, USA). To assay for TfR internalization, the medium was removed, the cells were rinsed twice with Eagle's minimum essential medium (EMEM) and then incubated at $37^{\circ} \mathrm{C}$ for $45 \mathrm{~min}$ with $20 \mu \mathrm{g} / \mathrm{mL}$ AxTf before being rinsed twice with PBS. This allows AxTf time to bind to its receptor, internalize and traffic to the $\operatorname{ERC}(9,11,19)$. AxTf in the ERC appears as a bright red, fluorescent spot adjacent to each nucleus. The wells to be analyzed for TfR internalization were fixed at room temperature for $30 \mathrm{~min}$ with $100 \mu \mathrm{L} /$ well of $3.7 \%$ formaldehyde solution that also had $10 \mu \mathrm{g} / \mathrm{mL}$ Hoechst 33342, the nucleic acid stain. The Hoechst-labeled nuclei emit blue fluorescence and are used to count all the cells. To assay for TfR recycling after AxTf incubation and removal, the wells were incubated at $37^{\circ} \mathrm{C}$ for 60 min with EMEM containing $10 \%$ FBS. The cells were then rinsed with PBS and fixed as described earlier. Where indicated, the cells were incubated concurrently with AxTf and 250-400 $\mu \mathrm{M}$ monodansyl cadaverine.

Assays on HEK293 cells expressing a PTHR, conjugated by way of its C-terminal cytoplasmic tail to a GFP, have been described elsewhere (2). These cells were plated in 96-well, optically clear-bottomed microplates at a density of $10^{4}$ cells/ well in HEPES-buffered DMEM/F12 medium with $10 \%$ fetal bovine serum and $1 \%$ penicillin/streptomycin, two days before the experiment. For the experiments, the cells were treated with a 100-nM parathyroid hormone (PTH) solution in the same medium at $22^{\circ} \mathrm{C}$ for $2 \mathrm{~h}$ before fixing with the formaldehyde/Hoechst solution for $10 \mathrm{~min}$, followed by rinses.

For sample analysis, prepared plates were loaded onto the ArrayScan II System. Using the Receptor Internalization and Trafficking application, the ArrayScan II automatically read and analyzed the images collected from multiple fluorescent channels. In each well, cell fields were automatically located, focused on and exposed according to user-defined and application-specific criteria. Multiple channels enabled simultaneous quantification of multiple targets in the same cell, field of cells or a subpopulation within cell fields, specifically the Hoechst-labeled nuclei, the PTH receptor and the AxTf in the blue, green and red channels, respectively.

One field per well was imaged using a $10 \times$ objective lens with an average of 350 cells detected per field. Approximately $20 \mathrm{~min}$ were required to image and analyze a 96-well plate. For each well, the Receptor Internalization and Trafficking application reported (i) the number of nuclei (i.e., cells) and their area; (ii) the number and percentage of cells with fluorescently labeled receptors in the ERC; (iii) the average and the total areas of the ERC spots; and (iv) the average and total integrated intensities of these spots. The fluorescent ERC spots containing internalized receptors were identified by their intensity, area and shape. Further details on the image analysis and use of the ArrayScan System are described elsewhere $(2,5,12)$. After scanning, the plate data were reviewed and exported to an Excel $^{\circledR}$ spreadsheet using the Cellomics ${ }^{\mathrm{TM}}$ Data Viewer software. The image data were also saved and used to corroborate the numerical data.

\section{RESULTS}

Figure 1, B and C, show the internalization and recycling of the TfR, respectively, illustrating the utility of fluorescently labeled Tf as a marker for its receptor's internalization and recycling. Bright peri-nuclear staining, typical of internalized receptor in ERC, was observed (Figure 1B). Faint staining of recycled receptors (Figure 1C) was observed following successful incubation of cells in nonfluorescent chase medium.

Figure 2A shows the results from a 96-well plate in which half the wells (columns 7-12) contained cells with internal-

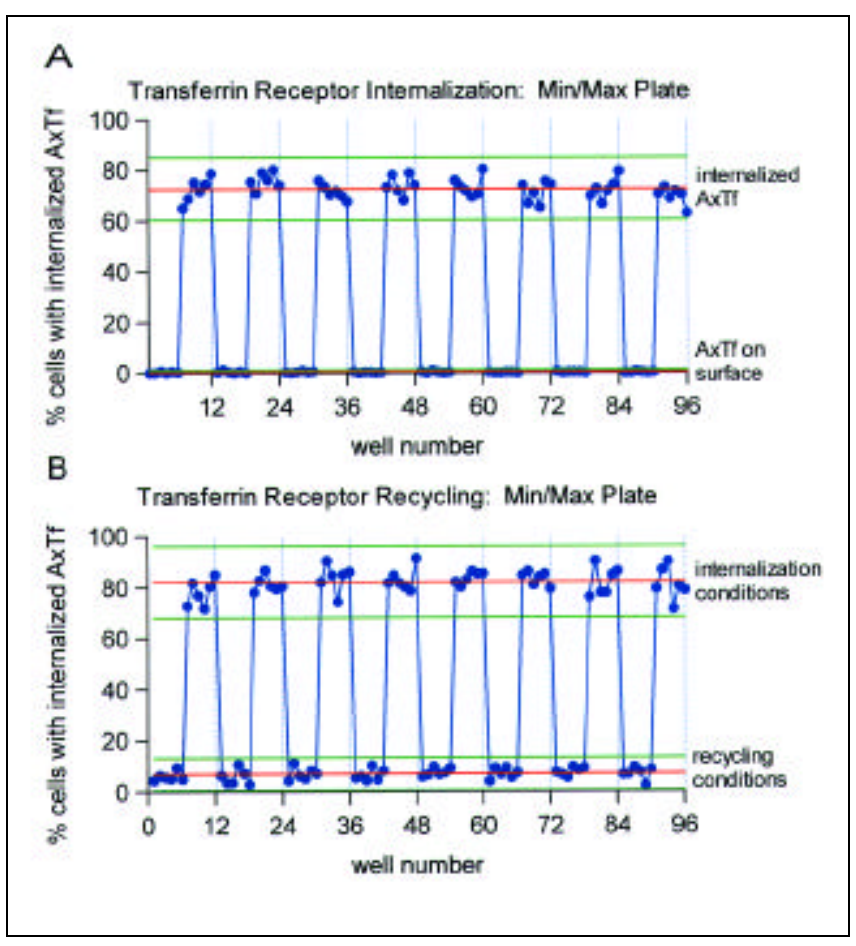

Figure 2. TfR internalization and recycling quantification. (A) Results from a 96-well plate in which internalization was allowed to occur in half the wells by incubating them with $20 \mu \mathrm{g} / \mathrm{mL}$ AxTf for $45 \mathrm{~min}$ at $37^{\circ} \mathrm{C}$. Internalization was blocked and AxTf remained on the cell surface in the remaining wells by incubating them with AxTf at $4^{\circ} \mathrm{C}$. (B) Results from a plate in which internalization was allowed to occur in all the wells as just described, but then half the wells were fixed (internalization conditions), and the remaining wells were incubated in fresh, nonfluorescent chase media for an additional $60 \mathrm{~min}$ to measure receptor recycling. In this time, most of the TfR exited the ERC, resulting in little fluorescence remaining. Both plates were imaged and analyzed on the ArrayScan II. Plotted is the percentage of cells with internalized AxTf for each well. The mean maximum and minimum values are shown by the red line, and the 99 percentiles are shown by the green lines. To determine how well an assay performed, the signal-to-noise ratio, defined as ,

$$
s / N=\frac{\left|\mu_{c+}-\mu_{c-}\right|}{\sqrt{\sigma_{c+}^{2}+\sigma_{c-}^{2}}}, \mid
$$

and the Z-factor, defined as

$$
Z=1-\frac{\left(3 \sigma_{c_{+}}+3 \sigma_{c_{-}}\right)}{\left|\mu_{c+}-\mu_{c-}\right|} \mid
$$

were calculated, where the maximum signal wells give a mean result of $\mu_{\mathrm{C}+}$ with an SD $\sigma_{\mathrm{C}+}$, and the minimum signal wells give a mean result of $\mu_{\mathrm{C} \text { - with }}$ an SD $\sigma_{\mathrm{C}-}$. 
ized AxTf, and the remaining wells (columns 1-6) contained AxTf that did not internalize but remained on the surface. The surface-bound, noninternalized condition was achieved by incubating the cells with AxTf at $4^{\circ} \mathrm{C}$ instead of $37^{\circ} \mathrm{C}$. The percentage of cells with internalized AxTf is plotted for each well (Figure 2A), and the mean and SD was $72.6 \pm 4.1$ for the positive wells (internalization) and $0.3 \pm 0.3$ for the negative wells (no internalization). These results give a high signal-tonoise ratio (17.6) between the internalized and noninternalized conditions; thus, this internalization assay provides a significant window between the positive and negative conditions to allow for screening.

Figure 2B shows the results from a plate in which half the wells were treated for AxTf internalization (columns 7-12) and the rest were treated for AxTf recycling (columns 1-6). Under recycling conditions, little staining was observed, which was consistent with successful TfR recycling from the ERCs (Figure 1C). The mean percentage of cells containing internalized AxTf was $82.1 \pm 4.7$, compared to $6.9 \pm 2.1$ for the recycled wells, giving a signal-to-noise ratio of 14.6.

To evaluate these assays for their suitability for screening and to compare them to other assays, we calculated their Zfactor (28). This dimensionless statistical parameter is used to evaluate an assay for its suitability for screening and to compare it to other assays. The results shown in Figure 2 gave Zfactors of 0.82 and 0.73 , confirming that these are excellent assays for screening, with large separation bands between the negative and positive responses (28). An AxTf internalization assay validation was performed in which two plates were prepared for maximum internalization, two prepared for minimum internalization, and two made for half-maximal and half-minimal internalization on three separate days with the TfR HitKit and run on the ArrayScan II System. This validation gave a mean Z-factor of $0.73 \pm 0.12$, a mean signal-tonoise ratio of $13.3 \pm 4.0$ and a maximum signal plate well variation of less than $10 \%$. The high signal-to-noise and Z-

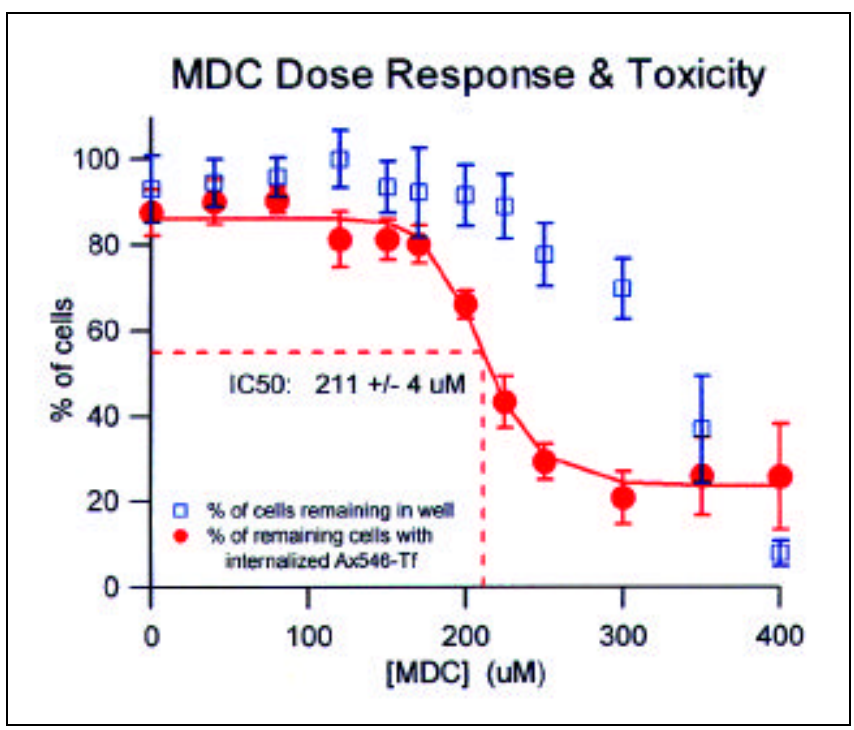

Figure 3. MDC dose response. To determine the $\mathrm{IC}_{50}$ of MDC to inhibit TfR internalization, cells were co-incubated with AxTf and increasing concentrations of MDC for $45 \mathrm{~min}$ before fixation and analysis on the ArrayScan II system. The percentage of cells with internalized AxTf is plotted (circles). High MDC concentrations are toxic and cause cell loss. Thus, the percentage of cells remaining in the well was also plotted (squares). Each concentration of MDC was applied to six wells, and their mean value and SD are plotted. factors, plus the low variability between wells, indicate this is a robust assay for screening.

The transglutaminase inhibitor, monodansyl cadaverine (MDC), impedes internalization and trafficking to the ERC (4) and was used as a demonstration compound. To measure MDC's $\mathrm{IC}_{50}$ concentration for TfR internalization, cells were co-incubated with AxTf and different concentrations of MDC and then treated as before and scanned on the ArrayScan II System. The percentage of cells with internalized AxTf was plotted (Figure 3 ), giving an $\mathrm{IC}_{50}$ value of $211 \pm 4 \mu \mathrm{M}$ within the range of other reports $(1,4,22-24,26)$. MDC can be toxic and cause cell loss. The ArrayScan II System simultaneously counts the number of cells remaining, which is used as a measure of toxicity (Figure 3). Other studies that used MDC did not account for its toxic effects at high doses, which likely contributes to the variability in reported $\mathrm{IC}_{50}$ values $(1,4$, 22-24,26). This demonstrates that the Receptor Internalization and Trafficking application on the ArrayScan II can be used to determine compound dose-response curves, toxicity and $\mathrm{IC}_{50}$ or $\mathrm{EC}_{50}$ values.

The Receptor Internalization and Trafficking application can also quantify the association of other receptors with the ERC. The presence of a GPCR in the ERC indicates it has been stimulated by an agonist, and quantifying it is a method to assay for GPCR activation. To demonstrate this, a physiologically functional GFP conjugate of the PTHR expressed in HEK293 cells was used (2). In this assay, the majority of the GFP-PTHR was on the cell surface and gave a diffuse staining pattern. When the cells were treated with PTH, GFP-PTHR internalization and trafficking to the ERC occurred, resulting in

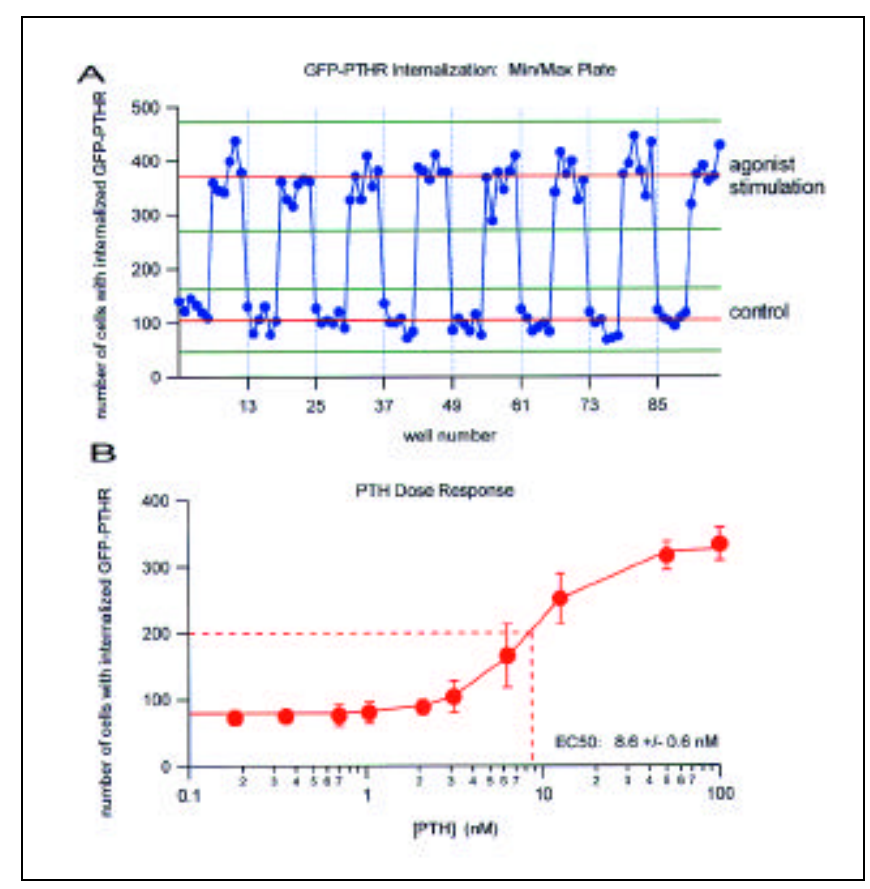

Figure 4. PTHR internalization quantification and PTH dose response. (A) Representative plate showing magnitude of PTHR internalization where half the wells of a 96-well plate were treated with PTH (100 nM PTH, $2 \mathrm{~h}$ ). (B) PTH dose response curve. To determine the $\mathrm{EC}_{50}$ of PTH, the wells in each column of a 96-well plate containing HEK293 cells expressing the PTHR-GFP were treated with different concentrations of PTH for $2 \mathrm{~h}$. For both experiments following PTH treatment, the cells were fixed and labeled with Hoechst 33342 and then the plate was imaged on the ArrayScan II. The response variable plotted is the mean number of cells with internalized PTHR detected for the wells. 
the typical discrete punctate peri-nuclear staining. Figure 4A depicts half a 96-well plate treated with PTH to induce internalization. The number of cells with internalized GFP-PTHR detected is plotted for each well. The signal-to-noise ratio between the stimulated and unstimulated wells was 6.9. Out of a total of 24 experiments (eight plates, each run on three different ArrayScan II instruments), the mean number of cells with internalized GFP-PTHR for the stimulated wells was $303.3 \pm$ 2.5 , compared to $74.7 \pm 0.7$ for the vehicle-treated wells; the mean signal-to-noise value was $5.3 \pm 0.9$. Further details of this screening assay have been reported elsewhere (2).

Figure 4B shows a dose-response curve for PTH stimulation, where PTHR-GFP expressing cells were treated with varying concentrations of PTH and then imaged on the ArrayScan II. The $\mathrm{EC}_{50}$ associated with PTHR internalization with this assay was $8.6 \pm 0.6 \mathrm{nM}$, which is in the same range as in other reports (2). GPCRs that do not internalize or are destined for degradation through the use of the lysosomal pathway will not be detected by this assay because the application is optimized to analyze internalized GPCRs located in a compact fluorescent peri-nuclear spot.

\section{DISCUSSION}

We demonstrate three quantitative HCS assays: GPCR internalization, TfR internalization and TfR recycling. The receptor internalization and trafficking application measures compound effects on the ligand-induced internalization and recycling of GPCRs, physiologically functional GPCR chimeras and non-GPCRs. Also, the assays distinguish and quantify agonist and antagonist responses, and determine their toxicity and effective concentration ranges. They demonstrate low-variability, high-dynamic ranges and high signal-to-noise ratios, forming the basis of a robust screening platform. Cellular activities such as intracellular trafficking that involve the redistribution of macromolecules within the cell are best quantified by high-resolution imaging on whole cells such as that achievable by the ArrayScan II System, rather than total intensity changes or population responses. Further, the fully automated multiplexing capability of the ArrayScan II System uniquely allows for the simultaneous monitoring of and crosscorrelation among multiple targets in the same cell. Examples include activation of several different GPCRs, second messengers and transcription factors, along with compound cytotoxicity information, defining Cellomics' High Content Screening approach to drug discovery.

\section{REFERENCES}

1.Chow, J.C., G. Condorelli and R.J. Smith. 1998. Insulin-like growth factor-I receptor internalization regulates signaling via the Shc/mitogen-activated protein kinase pathway, but not the insulin receptor substrate-1 pathway. J. Biol. Chem. 273:4672-4680.

2.Conway, B.R., L.K. Minor, J.Z. Xu, J.W. Gunnet, R. DeBiasio, M.R. D’Andrea, R. Rubin, R. DeBiasio et al. 1999. Quantification of G-protein coupled receptor internalization using G-protein coupled receptor-green fluorescent protein conjugates with the ArrayScan ${ }^{\mathrm{TM}}$ high-content screening system. J. Biomol. Screening 4:75-86.

3.Daro, E., P. van der Sluijs, T. Galli and I. Mellman. 1996. Rab4 and cellubrevin define different early endosome populations on the pathway of transferrin receptor recycling. Proc. Natl. Acad. Sci. USA 93:9559-9564.

4.Davies, P.J.A., D.R. Davies, A. Levitzki, F.R. Maxfield, P. Milhaud, M.C. Willingham and I.H. Pastan. 1980. Transglutaminase is essential in receptormediated endocytosis of alpha2-macroglobulin and polypeptide hormones. Nature 283:162-167.

5.Ding, G.J.F., P.A. Fischer, R.C. Boltz, J.A. Schmidt, J.J. Colaianne, A.
Gough, R.A. Rubin and D.K. Miller. 1998. Characterization and quantitation of NF-kappaB nuclear translocation induced by interleukin-1 and tumor necrosis factor-alpha. J. Biol. Chem. 273:28897-28905.

6.Drmota, T., G.W. Gould and G. Milligan. 1998. Real time visualization of agonist-mediated redistribution and internalization of a green fluorescent proteintagged form of the thyrotropin-releasing hormone receptor. J. Biol. Chem. 273:24000-24008.

7.Dunn, K.W., T.E. McGraw and F.R. Maxfield. 1989. Iterative fractionation of recycling receptors from lysosomally destined ligands in an early sorting endosome. J. Cell Biol. 109:3303-3314.

8.Galli, T., T. Chilcote, O. Mundigl, T. Binz, H. Niemann and P. De Camilli. 1994.Tetanus toxin-mediated cleavage of cellubrevin impairs exocytosis of transferrin receptor-containing vesicles in CHO cells. J. Cell Biol. 125:1015-1024.

9.Ghosh, R.N., D.L. Gelman and F.R. Maxfield. 1994. Quantification of low density lipoprotein and transferrin endocytic sorting HEp2 cells using confocal microscopy. J. Cell Sci. 107:2177-2189.

10.Ghosh, R.N., W.G. Mallet, T.T. Soe, T.E. McGraw and F.R. Maxfield. 1998. An endocytosed TGN38 chimeric protein is delivered to the TGN after trafficking through the endocytic recycling compartment in CHO cells. J. Cell Biol. 142:923-936.

11.Ghosh, R.N. and F.R. Maxfield. 1995. Evidence for nonvectorial, retrograde transferrin trafficking in the early endosomes of HEp2 cells. J. Cell Biol. 128:549-561.

12.Giuliano, K.A., R.L. DeBiasio, R.T. Dunlay, A. Gough, J.M. Volosky, J. Zock, G N. Pavlakis and D.L. Taylor. 1997. High-content screening: a new approach to easing key bottlenecks in the drug discovery process. J. Biomol. Screening 2:249-259.

13.Giuliano, K.A. and D.L. Taylor. 1998. Fluorescent-protein biosensors: new tools for drug discovery. Trends Biotechnol. 16:99-146.

14.Gruenberg, J. and F.R. Maxfield. 1995. Membrane transport in the endocytic pathway. Curr. Opin. Cell Biol. 7:552-563.

15.Johnson, A.O., R.N. Ghosh, K.W. Dunn, R. Garippa, J. Park, S. Mayor, F.R. Maxfield and T.E. McGraw. 1996. Transferrin receptor containing the SDYQRL motif of TGN38 causes a reorganization of the recycling compartment but is not targeted to the TGN. J. Cell Biol. 135:1749-1762.

16.Kallal, L., A.W. Gagnon, R.B. Penn and J.L. Benovic. 1998. Visualization of agonist-induced sequestration and down-regulation of a green fluorescent protein-tagged beta2-adrenergic receptor. J. Biol. Chem. 273:322-328.

17.Mayor, S., J.F. Presley and F.R. Maxfield. 1993. Sorting of membrane components from endosomes and subsequent recycling to the cell surface occurs by a bulk flow process. J. Cell Biol. 121:1257-1269.

18.Mayor, S., S. Sabharanjak and F.R. Maxfield. Cholesterol-dependent retention of GPI-anchored proteins in endosomes. EMBO J. 17:4626-4638.

19.Mukherjee, S., R.N. Ghosh and F.R. Maxfield. 1997. Endocytosis. Physiol. Rev. 77:759-803.

20.Mukherjee, S., T.T. Soe and F.R. Maxfield. 1999. Endocytic sorting of lipid analogues differing solely in the chemistry of their hydrophobic tails. J. Cell Biol. 144:1271-1312.

21.Mukherjee, S., X. Zha, I. Tabas and F.R. Maxfield. 1998. Cholesterol distribution in living cells: fluorescence imaging using dehydroergosterol as a fluorescent cholesterol analog. Biophys. J. 75:1915-1925.

22.Pierce, K.L., S. Maudsley, Y. Daaka, L.M. Luttrell and R.J. Lefkowitz. 2000. Role of endocytosis in the activation of the extracellular signal-regulated kinase cascade by sequestering and nonsequestering $\mathrm{G}$ protein-coupled receptors. Proc. Natl. Acad. Sci. USA 97:1489-1494.

23.Ray, E. and A.K. Samanta. Receptor-mediated endocytosis of IL-8: a fluorescent microscopic evidence and implication of the process in ligand-induced biological response in human neutrophils. Cytokine 9:587-596.

24.Sai, Y., M. Kajita, I. Tamai, J. Wakama, T. Wakamiya and A. Tsuji. 1998. Adsorptive-mediated endocytosis of a basic peptide in enterocyte-like Caco-2 cells. Am. J. Physiol. 275:G514-G520.

25.Tarasova, N.I., R.H. Stauber, J.K. Choi, E.A. Hudson, G. Czerwinski, J.L. Miller, G.N. Pavlakis, C.J. Michejda and S.A. Wank. 1997. Visualization of $\mathrm{G}$ protein-coupled receptor trafficking with the aid of the green fluorescent protein. Endocytosis and recycling of cholecystokinin receptor type A. J. Biol. Chem. 272:14817-14824.

26. Tolleshaug, H., T. Berg and K. Holte. 1982. Effects of local anesthetics and related compounds on the endocytosis and catabolism of asialo-glycoproteins in isolated hepatocytes. Biochim. Biophys. Acta 714:114-121.

27.Ullrich, O., S. Reinsch, S. Urbe, M. Zerial and R.G. Parton. 1996. Rab11 regulates recycling through the pericentriolar recycling endosome. J. Cell Biol. 135:913-924.

28.Zhang, J.-H., T.D. Y. Chung and K.R. Oldenburg. A simple statistical parameter for use in evaluation and validation of high throughput screening assays. J. Biomol. Screening 4:67-73.

Address correspondence to Elizabeth S. Woo, Director, Technical Marketing, Cellomics ${ }^{\mathrm{TM}}$ Inc., 635 William Pitt Way, Pittsburgh,PA 15238,USA.e-mail: ewoo@cellomics.com 